\title{
Effect of Pressure Gradient on the Development of Görtler Vortices
}

\author{
Leandro Marochio Fernandes ${ }^{1, *}$, Marcio Teixeira de Mendonça²
}

How to cite

Fernandes LM (iD https: / / orcid. org/0000-0003-4965-7233

Mendonça M (D) T https: / / orcid.org/0000-0002-6630-0749

\begin{abstract}
Fernandes LM, Mendonça MT (2019) Effect of Pressure Gradient on the Development of Görtler Vortices. J Aerosp Technol Manag, 11: e4519. https://doi.org/10.5028/ jatm.v11.1076
\end{abstract}

\begin{abstract}
Boundary layers over concave surfaces may become unstable due to centrifugal instability that manifests itself as stationary streamwise counter rotating vortices. The centrifugal instability mechanism in boundary layers has been extensively studied and there is a large number of publications addressing different aspects of this problem. The results on the effect of pressure gradient show that favorable pressure gradients are stabilizing and adverse pressure gradient enhances the instability. The objective of the present investigation is to complement those works, looking particularly at the effect of pressure gradient on the stability diagram and on the determination of the spanwise wave number corresponding to the fastest growth. This study is based on the classic linear stability theory, where the parallel boundary layer approximation is assumed. Therefore, results are valid for Görtler numbers above 7, the lower limit where local mode linear stability analysis was identified in the literature as valid. For the base flow given by the Falkner-Skan solution, the linear stability equations are solved by a shooting method where the eigenvalues are the Görtler number, the spanwise wavenumber and the growth rate. The results show stabilization due to favorable pressure gradient as the constant amplification rate curves are displaced to higher Görtler numbers, with the opposite effect for adverse pressure gradient. Results previously unavailable in the literature identifying the fastest growing mode spanwise wavelength for a range of Falkner-Skan acceleration parameters are presented.
\end{abstract}

KEYWORDS: Götler vortices, Boundary layer, Pressure gradient, Linear stability analysis, Centrifugal instability.

\section{INTRODUCTION}

There is a large body of publications addressing centrifugal instability of boundary layers over concave surfaces. Centrifugal instability in boundary layers results in the development of counter rotating vortices known as Görtler vortices. The review papers by Floryan (1991) and Saric (1994) describe the main conclusions about this subject. Many effects that affect the development of Görtler vortices have been studied in the last two decades (Goulpié et al. 1996; Zhang and Fasel 1999; Souza 2003; Mitsudharmadi et al. 2006; Schrader et al. 2011; Sescu and Thompson 2015). These studies address effects such as compressibility, surface roughness, non-linearity and secondary instability, among others. Görtler vortices development in boundary layers with pressure gradient is also under study since the late 1990s. A detailed review can be found in Rogenski (2015), who used direct numerical simulation to study nonlinear effects in the development of Görtler vortices on boundary layers with constant pressure gradient and linear varying pressure gradient.

The study of Ragab and Nayfeh (1981) was one of the first studies to consider the effect of pressure gradient on the development of Görtler vortices. Besides the effect of pressure gradient, they also studied the effect of the displacement thickness on the base

\footnotetext{
1.Instituto Federal de Educação, Ciência e Tecnologia do Espírito Santo - Campus Cachoeiro de Itapemirim - Engenharia Mecânica - Cachoeiro de Itapemirim/ES - Brazil. 2.Departamento de Ciência e Tecnologia Aeroespacial - Instituto de Aeronáutica e Espaço - Divisão de Propulsão Aeronáutica - São José dos Campos/SP - Brazil. 
flow, and showed that it significantly changes the stability results even for the Blasius boundary layer profile. Ragab and Nayfeh (1981) present neutral stability curves for Falkner-Skan type boundary layers with pressure gradient. Their results show that favorable pressure gradients are stabilizing while adverse pressure gradients are destabilizing, especially for low wavenumbers. For large values of the acceleration parameters, all wavenumbers bellow a certain limit seem to be unstable. Ragab and Nayfeh (1981) also studied the stability of boundary layers of variable curvature walls, computing the boundary layer velocity profiles with a marching scheme. The authors show that the results are different than those obtained with a local similarity solution due to the choice of initial conditions, especially in favorable pressure gradient regions.

The few results presented by Ragab and Nayfeh (1981) for variable wall curvature boundary layers are significant for flows over airfoils, as shown by the work of Mangalam et al. (1985). They performed experimental measurements on the concave surface of an airfoil, but no details of the pressure gradient distribution were given. Their results show that, as for constant free stream velocity and constant pressure gradient, once the spanwise wavenumber is established they remain fixed downstream up to the nonlinear and saturation region.

In 1994, Finnis and Brown (1994) presented another experimental study of the effect of pressure gradient on the development of Görtler vortices. They considered only favorable pressure gradient and showed its stabilizing effect as well as the fact that the spanwise wavenumber does not change due to the flow acceleration. Comparisons of experimental results and linear stability theory results for the streamwise velocity component were made, showing that for the range of parameters considered, the linear stability theory results provide a good approximation. A comparison between the local linear stability theory and a streamwise marching technique was presented by Goulpié et al. (1996). They considered base flows given by the Falkner-Skan solution and confirmed previous results, where favorable pressure gradient are stabilizing and adverse pressure gradient are destabilizing. Their study was motivated by the work of Otto and Denier (1993), who reported a stabilizing effect of adverse pressure gradient on the development of Görtler vortices computed with a marching analysis. Goulpié et al.'s conclusion was that the marching technique depends on the starting computation position downstream of the leading edge of the plate and on the proper choice of initial conditions, which may only be correctly specified after a receptivity analysis. The results of a marching analysis are also dependent on the criterion used to define the neutral point. Therefore, the concept of a single neutral curve is not tenable. Based on the disputed validity of the linear stability theory for low spanwise wavenumbers, they question the conclusion that Görtler vortices below a certain wavenumber are always unstable, as inferred by Ragab and Nayfeh (1981).

Non-parallel effects can be accounted for using series expansion in terms of powers of one over the square of the Reynolds number for certain specific cases, as done by Itoh (2001) for the stagnation point flow. The formulation results in a critical Görtler number for a finite value of the spanwise wavenumber which is accurate from the solution of the lowest order expansion even for relatively low Reynolds numbers.

With an interest in flow control, the combined effect of pressure gradient and suction or blowing was studied by Matsson (2008) using a non-linear, non-local downstream marching model. He used the saturation amplitude as an indication of stabilization by favorable pressure gradient and suction. Both concave and convex walls can be unstable for adverse pressure gradient if a base flow has an overshoot in the velocity profile. The base flow considered by Matsson (2008) was of the Falkner-Skan family including cases with overshoot similar to wall jet flows. Pressure gradient and suction and blowing change not only the boundary layer thickness, but also the lateral spreading of the characteristic mushroom-like structures of the Görtler vortices. These changes in topology are accompanied by changes in the saturation amplitude and growth rates and after saturation the amplitude decays to a lower constant level, followed by secondary instability. Matsson's results also show that favorable pressure gradient results in stronger vortices in the nonlinear region. The vortices grow stronger with higher kinetic energy in the nonlinear region, and maintain their structure further downstream before saturation. In other words, the vortices strength increases, but not their instability, as they remain coherent further downstream. Matsson (2008) associated this higher kinetic energy with instability, but in reality, maintaining the vortical structure without saturation or breakdown for a longer streamwise extent is actually associated with higher stability of the mushroom structure (secondary instability). Adverse pressure gradient results are more unstable on the linear region, saturate earlier and at a lower amplitude compared to the Blasius results. These results depend also on the spanwise wavenumber, but only two wavenumbers were tested by the author. Taking the Blasius boundary layer solution as reference, the 
smaller wavenumber $(\beta=0.22)$ chosen by Matsson $(2008)$ is more unstable than their higher wavenumber $(\beta=0.52)$, showing that the former is closer to the wavenumber associated with the maximum amplification. That pattern was the same for either suction/ blowing and pressure gradient, in a sense that suction and favorable pressure gradient are stabilizing. The greatest stabilization was obtained with both suction and favorable pressure gradient.

The results for the nonlinear development and saturation of Görtler vortices obtained by Matsson (2008) can be compared with the results obtained by Souza and his group (Rogenski et al. 2013; 2016a; 2016b), who have been using direct numerical simulation (DNS) to study the development of Görtler vortices in boundary layers with pressure gradient. In a first paper Rogenski et al. (2013) studied the effect of curvature variation on the nonlinear development of Görtler vortices as a way to control their growth and consequent transition to turbulence. They show the stabilizing effect of a convex surface downstream of the concave wall that generates the primary counter rotating vortices. For a concave surface followed by a flat wall the results show the slow decay rate of the vortices on the flat wall region.

In a second paper Rogenski et al. (2016a) address the question of variable pressure gradient, with more details about the free stream condition than that given by Mangalam et al. (1985). A zero pressure gradient boundary layer was used as reference condition and three conditions were studied, constant pressure gradient, constant Hartree parameter and linear varying pressure gradient. Both favorable and adverse pressure gradients were considered. The results show that the adverse pressure gradient destabilizes the flow. The growth rate for constant adverse pressure gradient is larger than the growth rate for constant Hartree parameter, which may be explained by the fact that a constant Hartree parameter corresponds to a decaying adverse pressure gradient downstream. For constant pressure gradient, the higher the pressure gradient the higher the growth rate, but the higher adverse pressure gradient Hartree parameter does not result in the highest amplification.

According to Rogenski et al. (2016a), the effect of pressure gradient depends on the vortices spanwise wavenumbers. By considering three different values of spanwise wavenumber they observed that large spanwise wavenumbers are more unstable when the pressure gradient is favorable. For adverse pressure gradient the opposite is true, small spanwise wavenumbers are more unstable. These results suggest that the fastest growing spanwise wavenumber changes with the pressure gradient. This is one of the investigations proposed in the present paper. The present investigation tries to clarify the effect of pressure gradient on the fastest growing mode in order to justify the stronger instability of the large spanwise wavenumber vortices in a favorable pressure gradient, confirm the explanation offered by Rogenski et al. (2016a) based on the size of the vortices, thickness of the shear layer and velocity gradients.

Rogenski et al. (2016a) also considered cases where the pressure gradient went from adverse to favorable and from favorable to adverse. Again, by considering the same three distinct values of spanwise wavenumbers, they show that vortices with different spanwise wavelength respond differently to pressure gradient, but regardless of the wavelength, going from favorable to adverse pressure gradient results in greater instability than going from adverse to favorable pressure gradient.

The non-linear regime was further explored by Rogenski et al. (2016b). They studied the development of Görtler vortices up to the nonlinear breakdown stage in boundary layers with pressure gradient. They use the energy of the fundamental Fourier mode and of the mean flow distortion as an indication of saturation to show that adverse pressure gradient anticipates saturation while favorable pressure gradient delays saturation. The energy at the saturation point is also higher for adverse pressure gradient. Three different spanwise wavenumbers were tested by Rogenski et al. (2016b), $\Lambda=160,305$ and 450. They show that the saturation point depends also on the wavenumber. For all spanwise wavenumbers tested, adverse pressure gradient promotes earlier saturation of the Görtler vortices, while favorable pressure gradient results in latter saturation and saturation at a lower kinetic energy level. Saturation takes place earlier for the lower spanwise wavenumber. The kinetic energy at the saturation point is higher for the larger spanwise wavelength, except for $\Lambda=450$, where the kinetic energy is lower than for $\Lambda=305$. The results also indicate a possible correlation between adverse pressure gradient and the development of secondary instability of the varicose type.

The objective of the present investigation is to extend previous results, undertaking a more detailed analysis on the effect of pressure gradient on the stability diagram and on the fastest growing spanwise wavenumber. Previous studies considered only a limited number of spanwise wavenumbers. The location of a given wavenumber with respect to the fastest growing wavenumber is an important aspect which has not been considered in previous works. Even the results from Goulpié et al. (1996) considered a 
limited number of $\Lambda$, and 210 is shown to be the fastest growing mode among the spanwise wavenumber tested, but no conclusion of the actual fastest mode can be drawn.

This study proposes to investigate the location of the fastest growing wavenumber for different pressure gradient conditions in order to create a map of growth rate versus pressure gradient that gives a more complete picture of the stability condition. The location of the neutral curve and the levels of growth rate will be used as references to define stabilization or destabilization due to pressure gradient when compared to the zero pressure gradient base flow.

The paper is organized such that the section on the methodology presents the formulation for the base flow and for the stability equations. The section on the results presents comparisons between neutral curves for different pressure gradient conditions, along with the stability diagrams, showing the displacement of the constant growth rate curves due to pressure gradient and the location of the fastest growing spanwise wavenumber. A summary of the findings and conclusions are presented in the last section.

\section{METHODOLOGY}

The stability of boundary layers over concave surfaces can be studied using linear stability, normal modes formulation. But this approach is not valid for low Görtler numbers, which is the nondimensional controlling parameter in centrifugal instability on boundary layers. That is so because the growth of the disturbances is of the same order as the growth of the boundary layer and the flow cannot be considered parallel. For Görtler numbers lower that 7 the problem is parabolic and upstream dependent as shown by Bottaro and Luchini (1999) and Hall (1983). Nevertheless, the local, normal modes approach is useful to undertake a parametric study and results are reliable to Görtler numbers higher than Go = 7, identified by Bottaro and Luchini (1999) as the value above which the local normal mode approach is valid. Therefore, the present investigation only considers neutral curves for Görtler numbers above this threshold and no attempt is made in order to define a critical Görtler number. Also, the concept of the fastest growing spanwise wavenumber has been validated by experimental results in quiet test facilities, where no preferred wavelength is excited (Bippes 1978; Winoto et al. 1979; Aihara and Koyama 1981; Mangalam et al. 1985).

\section{BASE FLOW}

In the present investigation the base flow will be given by the solution of the Falkner-Skan similarity profile for different values of the pressure gradient parameter $\beta_{f s}$. The choice for the base flow given by the Falkner-Skan boundary layer profile follows Floryan (1991). The author presented an order of magnitude analysis showing that, for a concave plate with radius of curvature much larger than the boundary layer thickness, at first order approximation the base flow is that given by the boundary layer equations without any curvature terms and that the curvature effects are present only on the stability equations.

The Falkner-Skan similarity requires that (Eq. 1)

$$
U_{e}=x^{m}
$$

The similarity transformation in the normal to the wall direction is (Eqs. 2 and 3)

$$
\begin{gathered}
\eta=\frac{y}{\delta}, \quad \delta=\sqrt{\frac{\nu x}{U_{e}}} \\
\frac{u}{U_{e}}=f(\eta)
\end{gathered}
$$

The choice of similarity transformation results in the following relation (Eq. 4) between the pressure gradient parameter $\beta_{f s}$ and the exponent $m$, 


$$
\beta_{f s}=m
$$

and the resulting ordinary differential equation is (Eq. 5)

$$
f^{\prime \prime \prime}+\frac{\beta_{f s}+1}{2} f f^{\prime \prime}+\beta_{f s}\left(1-f^{2}\right)=0
$$

This is the Falkner-Skan similarity equation written in the same form used by Goulpié et al. (1996). This equation is solved by classical shooting methods, using a fourth order Runge-Kutta method to integrate from the wall to a position far on the free stream.

The Boundary conditions for this equation are no slip and impermeability at the wall and imposed free stream velocity away from the wall.

\section{LOCAL, NORMAL MODES ANALYSIS}

The centrifugal stability equations can be derived by decomposing the flow velocities as a base component and a disturbance (Eq. 6) (Floryan 1991).

$$
u(x, y, z, t)=U(y)+u^{\prime}(x, y, z, t)
$$

Normal modes solutions are sought assuming that the disturbances may be represent as (Eq. 7)

$$
u^{\prime}(x, y, z, t)=\hat{u}(y) \exp (\alpha x+i \beta z)
$$

where $\alpha$ is the growth rate and $\beta$ is the spanwise wavenumber and $\hat{\mathrm{u}}$ is a complex amplitude.

Substitution of the decomposition and the normal mode solution Eq. 7 back in the incompressible Navier-Stokes equations, the following stability equations (Eqs. 8 and 9) are derived

$$
\begin{aligned}
v^{I V}+ & \left(2 U G o^{2} \beta^{2}+\alpha U_{x y}+\beta V_{x}\right) \hat{u}+\alpha U_{x} \hat{u}^{\prime}+\left(\alpha U \beta^{2}+\beta^{4}+\alpha U_{y y}+\beta^{2} V_{y}\right) \hat{v}^{\prime}- \\
& -\left(\alpha U+2 \beta^{2}+V_{y}\right) \hat{v}^{\prime \prime}-V \hat{v}^{\prime \prime \prime}=0
\end{aligned}
$$

and

$$
\hat{u}^{\prime \prime}-\left(U \alpha+\beta^{2}+U_{x}\right) \hat{u}-V \hat{u}^{\prime}-U_{y} \hat{v}=0
$$

where ' represents eigenfunction $u$ and $v$ derivation with respect to the normal direction; the subscripts $y$ and $x$ represent derivation of the base flow with respect to the corresponding normal and streamwise directions.

These equations are written in nondimensional form, using as reference length scale in the streamwise direction the distance from the leading edge $x$ and in the normal and spanwise directions the boundary layer thickness parameter $\delta$, as suggested by Floryan (1991), such that using subscript $d$ to indicate dimensional quantities (Eq. 10),

$$
\alpha=\alpha_{d} x, \quad \text { and } \quad \beta=\beta_{d} \delta
$$

The velocities are also scaled differently, with $U$ representing the free stream velocity away from the wall and $R e$ the Reynolds number defined in Eq. 11

$$
u=\frac{u_{d}}{U}, \quad v=\frac{v_{d}}{U} R e, \quad w=\frac{w_{d}}{U} R e .
$$

Choosing $\delta=\sqrt{\nu x_{d} / U}$ as the reference length scale in the spanwise and normal directions, the Görtler number is defined 
as (Eq. 12)

$$
G o^{2}=k \delta e^{2}, \quad R e=\frac{U \delta}{\nu} .
$$

The boundary conditions are no slip at the wall and exponential decay away from the wall (Eq. 13 and 14).

$$
\begin{aligned}
& u=v=v^{\prime}=0 \quad \text { em } \quad y=0, \\
& u, v \rightarrow 0 \quad \text { para } \quad y \rightarrow \infty .
\end{aligned}
$$

The problem posed by Eqs. 8 and 9 and by the boundary conditions above results in an eigenvalue problem where the dispersion relation is $f(G o, \beta, \alpha)=0$.

The nonparallel terms $U_{x}, U_{x y}, V, V_{x}$ and $V_{y}$ will be neglected. For accelerating or decelerating flows due to pressure gradient, the first order boundary layer solution normal velocity component $V$ grows or decays continuously outside the boundary layer. This is inconsistent with the hypothesis that the disturbance decays exponentially away from the wall when the base flow velocity components are constant. In order to avoid this problem, Ragab and Nayfeh (1981) have proposed an optimal coordinate system based on the streamlines of the potential flow, such that for large values of the normal coordinate the normal velocity tends to a constant. The present investigation neglect these nonparallel terms.

\section{RESULTS}

On the following sections the neutral curves for boundary layers with favorable and adverse pressure gradients are compared to the stability diagram for a Blasius boundary layer. The constant amplification curves are compared for different values of the Falkner-Skan acceleration parameter. Also, The position of the fastest growing spanwise wavenumber $\Lambda_{\max }$ on each diagram will show the relation between $\Lambda_{\max }$ and pressure gradient.

\section{NEUTRAL CURVES}

It is well known that adverse pressure gradients destabilize the boundary layer and favorable pressure gradients have stabilizing effect. Figure 1 shows a comparison between the neutral curves for different values of the Falkner-Skan acceleration parameter

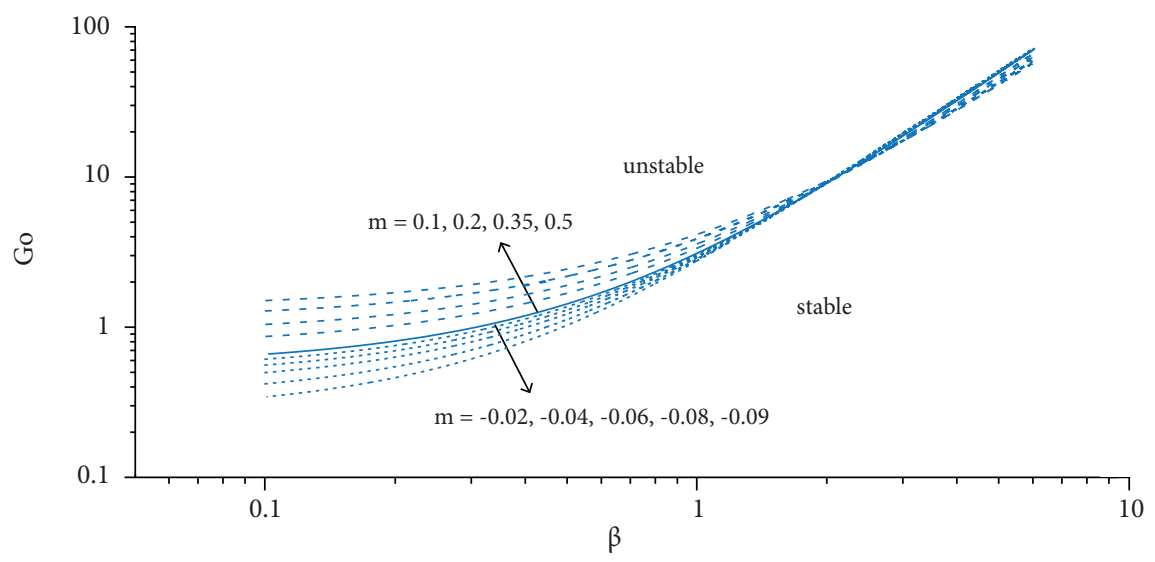

Figure 1. Comparison of neutral curves for different Falkner-Skan acceleration parameters. Solid line = Blasius boundary layer; dashed lines $=$ favorable pressure gradient; dotted lines = adverse pressure gradient. Above Go $\approx 10$ the instability region of the favorable pressure gradient flow is larger than that of the adverse pressure gradient flow. 
$\beta_{f s}$. Below $G o \approx 10$ the unstable region is reduced for favorable pressure gradient and increased for adverse pressure gradient. Above $G o \approx 10$ this trend is reversed, as seen in Fig. 2. This result was also reported by Goulpié et al. (1996), but one has to take into consideration that the position of the neutral curve is not the only aspect to take into account when judging the stability of a given flow. It is also needed to compare the growth rates and the most dangerous mode amplification.

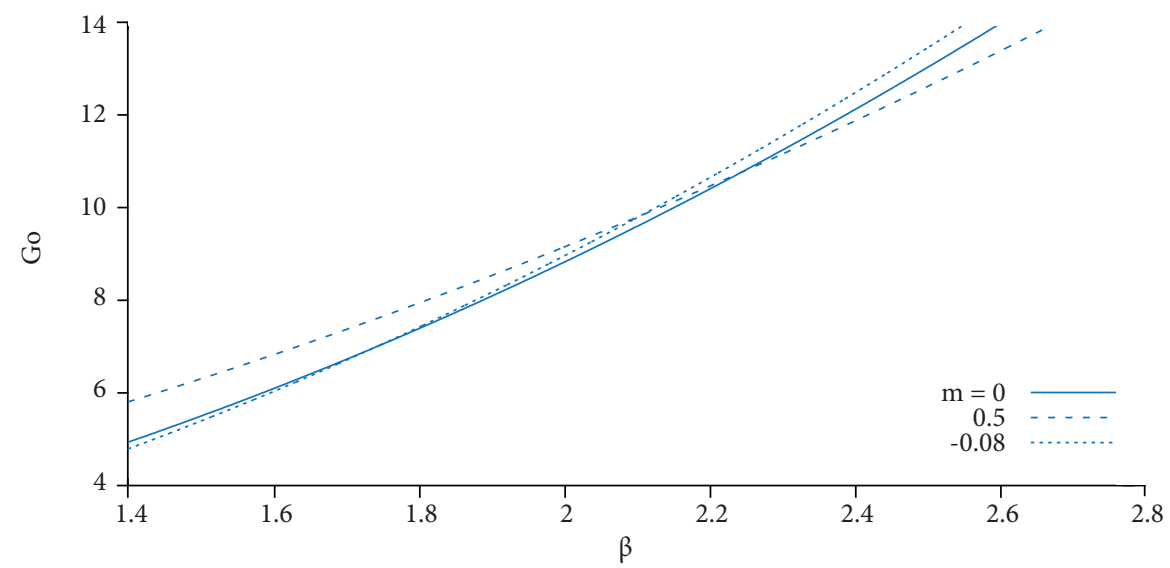

Figure 2. Comparison of neutral curves for different Falkner-Skan acceleration parameters. Detail of Fig. 1 in the range where the favorable pressure gradient has a larger range of unstable spanwise wavenumbers $\beta$ than the adverser pressure gradient.

\section{STABILITY DIAGRAMS}

The following results show the stability diagrams including lines of constant amplification $\alpha$ in order to evaluate the effect of pressure gradient. The reference case is the stability diagram for the Blasius boundary layer shown if Fig. 3.

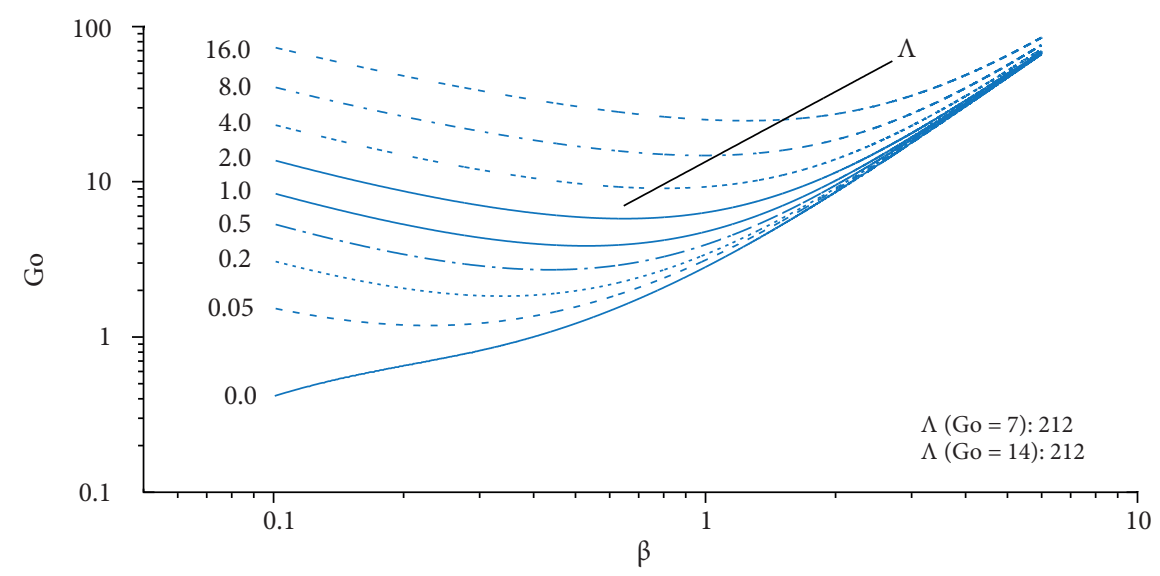

Figure 3. Görtler vortices stability diagram, zero pressure gradient boundary layer.

The eigenvalue solution stability diagrams presented in Figs. 3 to 12 show constant amplification rate curves for different values of $\alpha$ in the Go, $\beta$ plane. The straight lines correspond to the fastest growing spanwise wavenumber. For the Blasius boundary layer the non-dimensional spanwise wavenumber of the fastest growing mode is $\Lambda=212$ according to the criterion adopted here, where (Eq. 15)

$$
\Lambda=\frac{U \lambda}{\nu} \sqrt{k \lambda}=G_{o}\left(\frac{2 \pi}{\beta}\right)^{3 / 2}
$$

and $\lambda=2 \pi / \beta$ is the dimensional spanwise wavelength. 


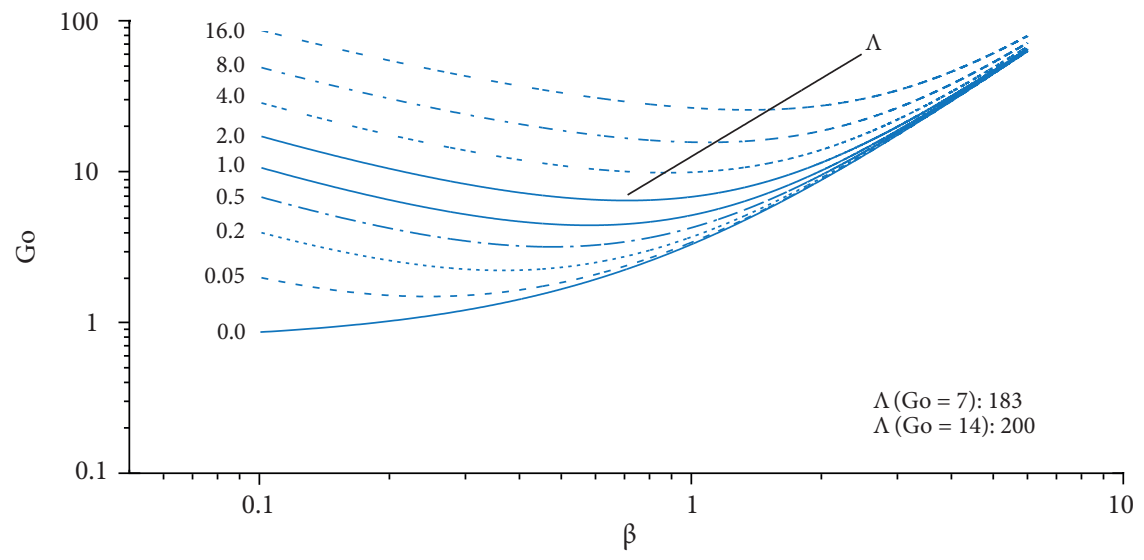

Figure 4. Görtler vortices stability diagram, Falkner-Skan boundary layer with $\beta_{f s}=0.1$.

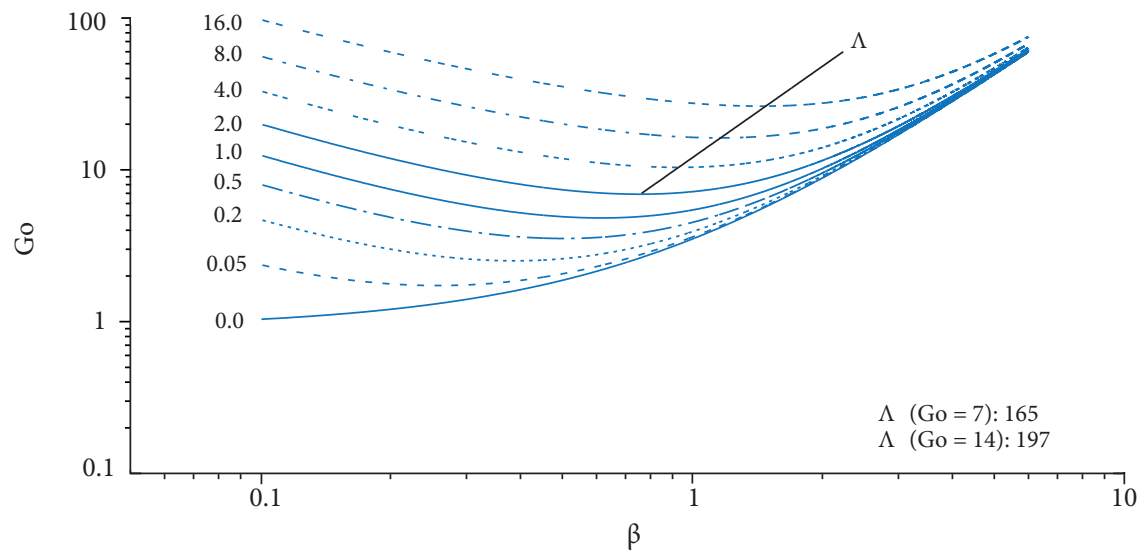

Figure 5. Görtler vortices stability diagram, Falkner-Skan boundary layer with $\beta_{f s}=0.2$.

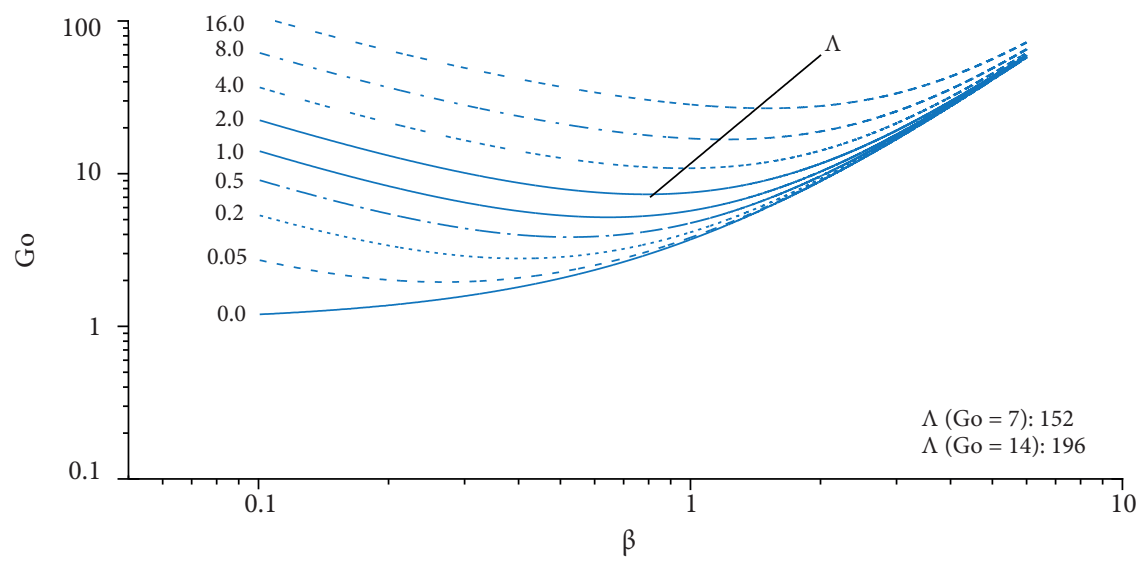

Figure 6. Görtler vortices stability diagram, Falkner-Skan boundary layer with $\beta_{f s}=0.3$. 


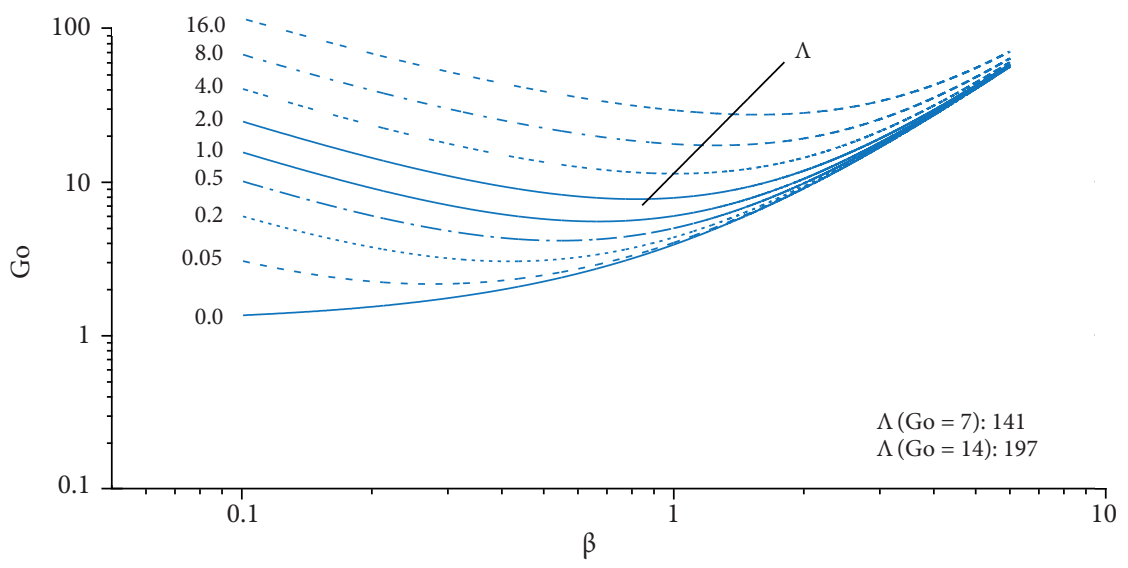

Figure 7. Görtler vortices stability diagram, Falkner-Skan boundary layer with $\beta_{f s}=0.4$.

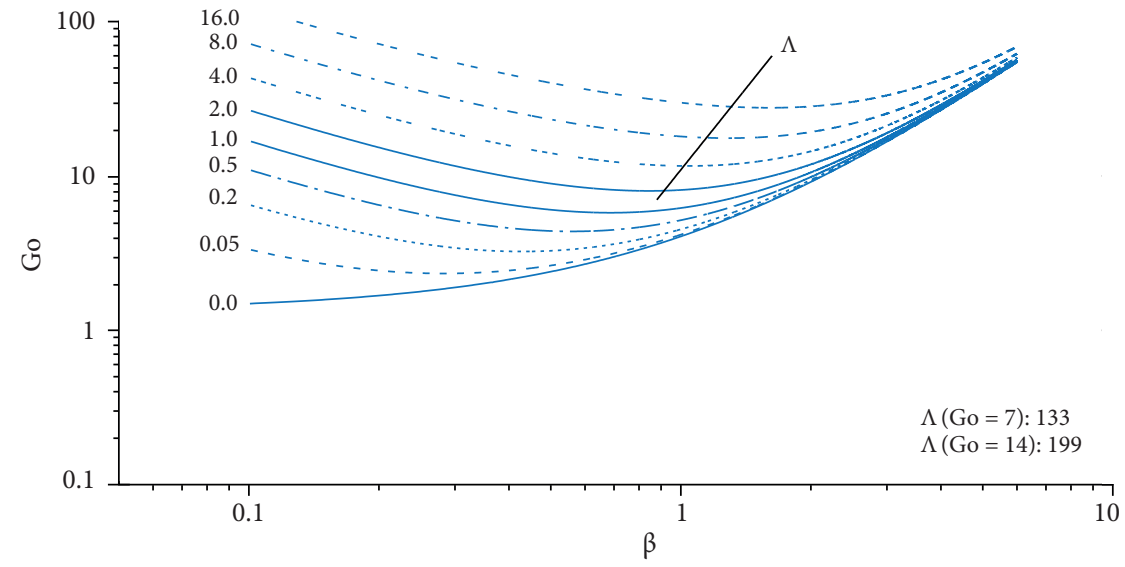

Figure 8. Görtler vortices stability diagram, Falkner-Skan boundary layer with $\beta_{f s}=0.5$.

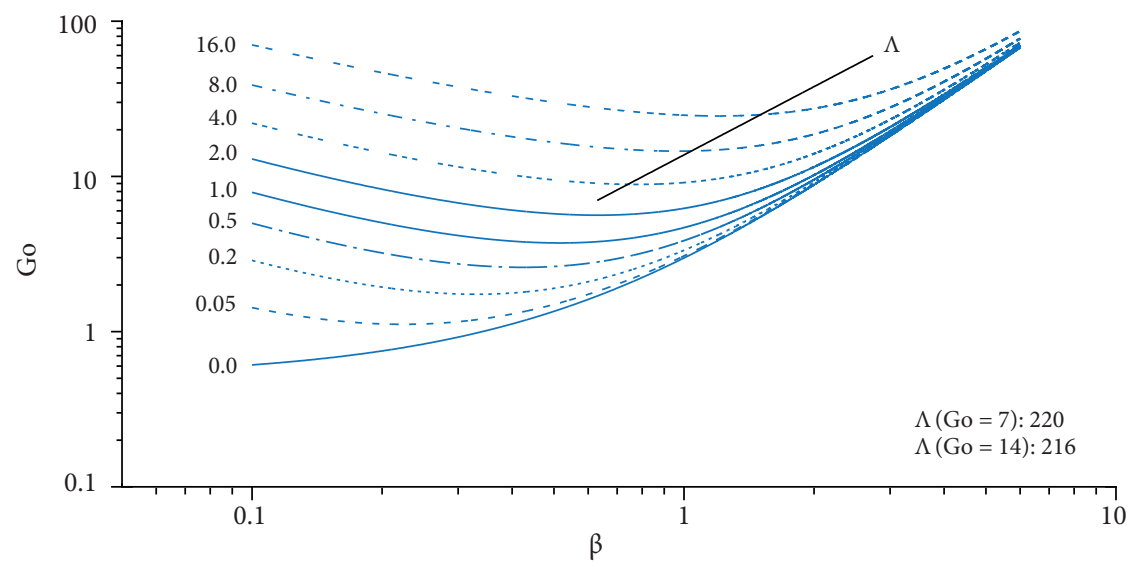

Figure 9. Görtler vortices stability diagram, Falkner-Skan boundary layer with $\beta_{f s}=-0.02$. 


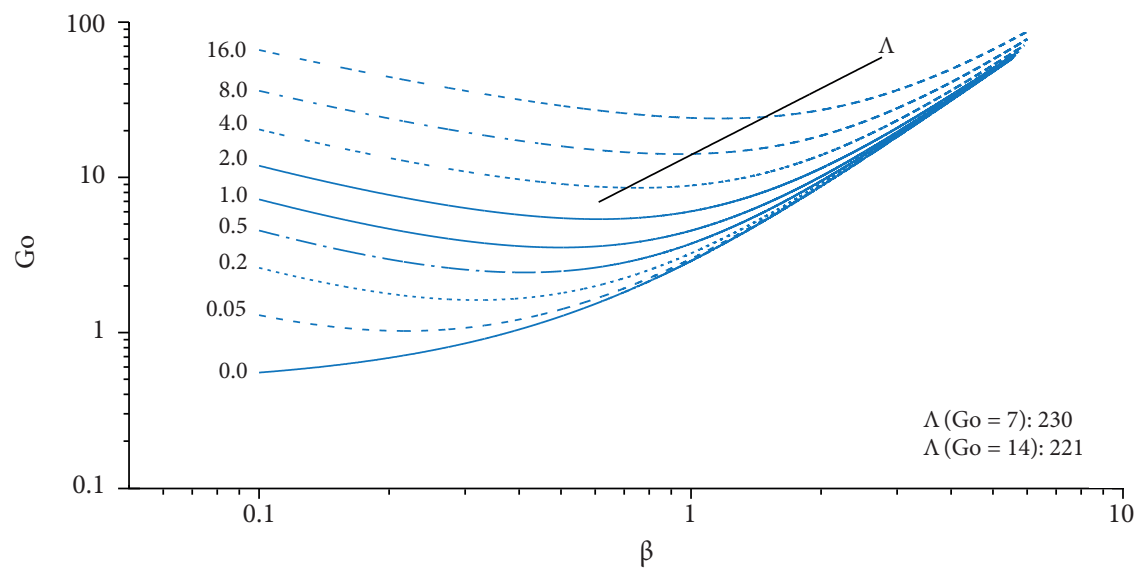

Figure 10. Görtler vortices stability diagram, Falkner-Skan boundary layer with $\beta_{f s}=-0.041$

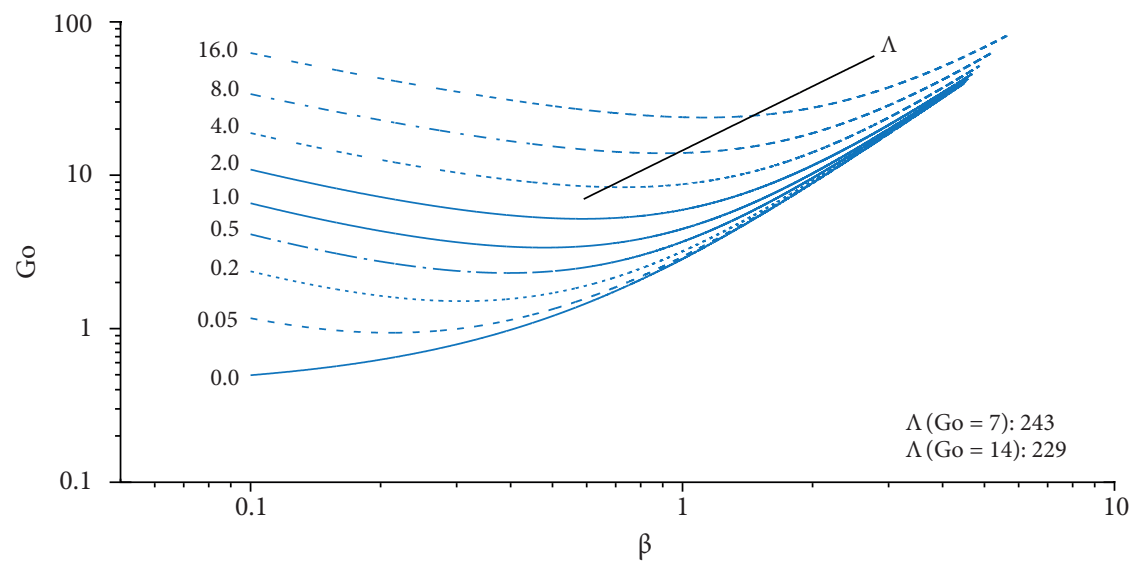

Figure 11. Görtler vortices stability diagram, Falkner-Skan boundary layer with $\beta_{f s}=-0.06$.

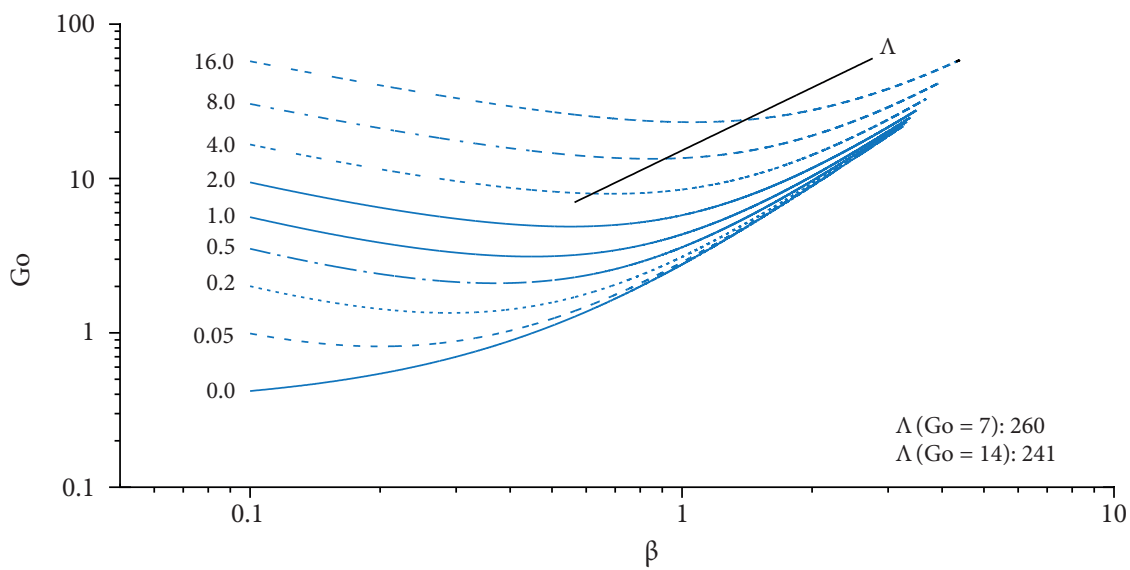

Figure 12. Görtler vortices stability diagram, Falkner-Skan boundary layer with $\beta_{f s}=-0.08$. 
Since $U_{e}=U_{e}(x)$ for boundary layers with pressure gradient, unlike in the Blasius boundary layer, $\Lambda$ varies downstream even for walls with constant curvature $k$ and constant spanwise wavelength $\lambda$. Following a line of constant $\lambda$ corresponds to the following variations of $G o, \beta$ and $\Lambda$ from a given starting position indicated by the subscript $i$, according to the following relations (Eqs. 16 to 18).

$$
\begin{aligned}
\frac{\beta}{\beta_{i}} & =\left(\frac{x}{x_{i}}\right)^{(1-m) / 2} \\
\frac{G o}{G o_{i}} & =\left(\frac{\beta}{\beta_{i}}\right)^{(3+m) /[2(1-m)]} \\
\frac{\Lambda}{\Lambda_{i}} & =\left(\frac{\beta}{\beta_{i}}\right)^{2 m /(1-m)}
\end{aligned}
$$

Two criteria were adopted to identify the fastest growing mode. One based on the spanwise wavenumber $\beta$ that has the largest growth rate at $G o=7$. The other one based on the largest amplification marching downstream from Go $=7$ to Go $=14$ along a path of constant spanwise wavelength $\lambda$. The amplification is computed according to Eq. 19.

$$
\ln \frac{A}{A_{0}}=\int_{x_{0}}^{x} \alpha d x
$$

The values for the fastest growing nondimensional wavenumber $\Lambda_{\max }$ depends on the choice for the downstream extent considered (in this case, $7<$ Go $<14$ ), because a line of constant $\lambda$ does no follow exactly the line of minimum Go for a given constant growth rate curve.

Figures 3 to 12 also show two values of $\Lambda$, one at the starting point $G o=7$ and one for the last streamwise position at $G o=14$, along the path of constant $\lambda$. For the Blasius boundary layer $\Lambda$ is constant according to Eq. 15.

The stability diagrams for favorable pressure gradients are presented in Figs. 4 to 8 . The lines of constant growth rate $\alpha$ are displaced upward as the acceleration parameter $\beta_{f_{s}}$ is increased. The value of $\Lambda$ for the fastest growing mode decreases as $\beta_{f s}$ increases, according to the criterion based on the amplification from $G o=7$ to $G o=14$. This result is shown in Table 1. The criterion based on the highest growth rate at $G o=7$ shows that $\Lambda_{\max }$ levels off after $\beta_{f s}=0.4$, increasing slowly afterward.

The opposite trend is observed for the adverse pressure gradient stability diagrams as shown if Figs. 9 to 12. Lines of constant growth rate $\alpha$ are displaced downward, indicating that higher amplification rates are reached earlier, at a lower Go. The fastest growing mode $\Lambda_{\max }$ increases continuously as summarized in Table 1.

Figures 13 and 14 show the variations the non-dimensional spanwise wavelength $\Lambda$ and the amplification rate $\alpha$ along the path of the fastest growing modes for different values of the Falkner-Skan acceleration parameter $\beta_{f s}$ including the values of the Blasius boundary layer. They complement and summarize the conclusions taken from the stability diagrams regarding the values of $\Lambda_{\max }$ and stability characteristics for different pressure gradient conditions. The growth rates increase continuously as the Falkner-Skan parameter goes from $\beta_{f s}=0.8$ to $\beta_{f s}=-0.8$

These results confirm previous results on the effect of pressure gradient on the stability of Görtler vortices, but cover a larger range of parameters. The non-dimensional spanwise wavenumber $\Lambda$ of the Blasius boundary layer associated with the fastest growing mode, reported in a range around $\Lambda_{\max }=210$, is often used as a reference for different studies. The present results show that $\Lambda_{\max }$ varies considerably with pressure gradient. The values of $\Lambda_{\max }$ for different values of the acceleration parameter $\beta_{f s}$ are reported. 
Table 1. Fastest growing mode spanwise wavenumber parameter $\Lambda$ for different Falkner-Skan acceleration parameter $\beta_{f s}$. Second and third columns integrated amplitude criterion. Forth column, maximum growth rate at Go $=7$ criterion.

\begin{tabular}{|c|c|c|c|c|c|c|c|}
\hline \multicolumn{4}{|c|}{ adverse $d p / d x$} & \multicolumn{4}{|c|}{ favorable $d p / d x$} \\
\hline$\beta_{f s}$ & $\Lambda_{i}\left(G_{0}=7\right)$ & $\Lambda_{f}[\mathrm{Go}=14]$ & $\Lambda$ & $\beta_{f s}$ & $\Lambda_{i}\left[G_{0}=7\right]$ & $\Lambda_{f}[\mathrm{Go}=14]$ & $\boldsymbol{\Lambda}$ \\
\hline 0.0 & 212 & 212 & 185 & 0.0 & 212 & 212 & 185 \\
\hline-0.01 & 216 & 214 & 187 & 0.1 & 183 & 200 & 172 \\
\hline-0.02 & 220 & 216 & 188 & 0.2 & 165 & 197 & 166 \\
\hline-0.03 & 225 & 219 & 191 & 0.3 & 152 & 196 & 163 \\
\hline-0.04 & 230 & 221 & 194 & 0.4 & 141 & 197 & 161 \\
\hline-0.05 & 236 & 225 & 197 & 0.5 & 133 & 199 & 161 \\
\hline-0.06 & 243 & 229 & 200 & 0.6 & 127 & 202 & 161 \\
\hline-0.07 & 251 & 235 & 205 & 0.7 & 121 & 206 & 161 \\
\hline-0.08 & 260 & 241 & 211 & 0.8 & 116 & 210 & 162 \\
\hline-0.09 & 275 & 253 & 220 & 0.9 & 112 & 212 & 164 \\
\hline
\end{tabular}

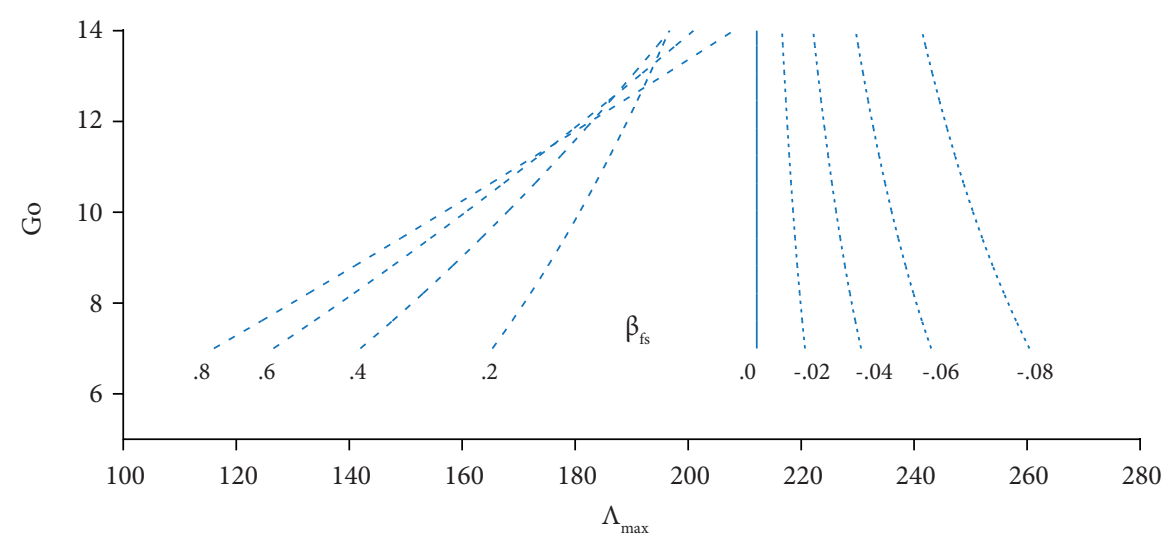

Figure 13. Variation of $\Lambda$ along the path corresponding to the fastest growing wavelength. Favorable and adverse pressure gradients.

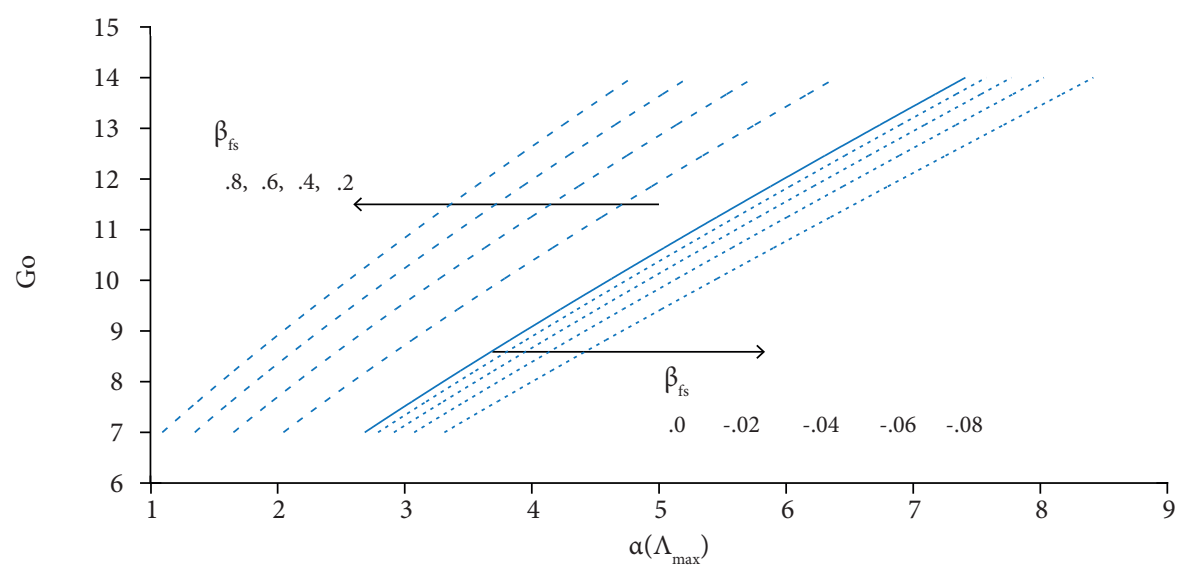

Figure 14. Variation of the growth rate $\alpha$ along the path corresponding to the fastest growing wavelength. Favorable and adverse pressure gradients. 


\section{CONCLUSIONS}

The present work presents further information on the effect of pressure gradient on the development of Görtler vortices in boundary layers over concave surfaces. The study was conducted based on the linear stability theory, which is valid for Görtler numbers above 7. Stability diagrams for values of the Falkner-Skan similarity solution acceleration parameter $\beta_{f s}$ ranging from favorable to adverse pressure gradients were compared to the stability diagram of the Blasius boundary layer. The results show the destabilizing effect of adverse pressure gradient and the stabilizing effect of favorable pressure gradient, as already reported in the literature. The constant growth rate curves are displaced upward or downward on the stability diagram depending on the pressure gradient, such that higher growth rates are encountered latter downstream or earlier upstream, respectively. The growth rates along the path in the streamwise direction are shown to increase with decreasing favorable pressure gradient or increasing adverse pressure gradient. The main focus of this work was on the determination of the fastest growing modes spanwise nondimensional wavenumber parameter $\Lambda_{\max }$. A range of Falkner-Skan acceleration parameters was investigated and the values of $\Lambda_{\max }$ were identified. The effect of pressure gradient on the development of Görtler vortices reported in previous investigations can be further understood when the relation between the reported wavelength on those works and the fastest growing mode for the given acceleration parameter is considered.

\section{AUTHOR'S CONTRIBUTION}

Conceptualization, Fernandes LM and Mendonça MT; Methodology, Fernandes LM and Mendonça MT; Investigation, Fernandes LM and Mendonça MT; Writing - Original Draft, Fernandes LM and Mendonça MT; Writing - Review and Editing, Fernandes LM and Mendonça MT; Funding Acquisition, Mendonça MT; Resources, Mendonça MT; Supervision, Mendonça MT.

\section{FUNDING}

There are no funders to report for this submission.

\section{REFERENCES}

Aihara Y, Koyama H (1981) Secondary instability of Görtler vortices: formation of periodic three-dimensional coherent structure. Transac Japan Soc Aeronautical and Space Science 24:78-94.

Bippes H (1978) Experimental study of the laminar-turbulent transition on a concave wall in a parallel flow. (TM 75243). NASA Technical Report. Bottaro A, Luchini P (1999) Görtler vortices: are they amenable to local eigenvalue analysis? Eur J Mech B/Fluids 18(1):47-65. https: // doi.org/10.1016/S0997-7546(99)80005-3

Finnis MV, Brown A (1994) The streamwise development of Görtler vortices in a favorable pressure gradient. Presented at: International Gas Turbine and Aeroengine Congress and Exposition; The Hague, Netherlands. https://doi.org/10.1115/94-GT-166

Floryan JM (1991) On the Görtler instability of boundary layers. Prog Aerospace Sci 28(3):235-271. https://doi.org/10.1016/03760421(91)90006-P

Goulpié P, Klingmann BGB, Bottaro A (1996) Görtler vortices in boundary layers with streamwise pressure gradient. Physics of Fluids 8:451-459. https://doi.org/10.1063/1.868799

Hall $P$ (1983) The linear development of Görtler vortices in growing boundary layers. J Fluid Mechanics 130:41-58. https://doi. org/10.1017/S0022112083000968

Itoh N (2001) A non-parallel theory for Görtler instability of falkner-skan boundary layers. Fluid Dynamics Research 28(5):383-396. https://doi.org/10.1016/S0169-5983(01)00006-5 
Mangalam SM, Dagenhart JR, Hepner TE, Meyers JF (1985) The Görtler instability on an airfoil. Presented at: 23rd AIAA Aerospace Sciences Meeting and Exhibit; Reno, USA. https://doi.org/10.2514/6.1985-491

Matsson OJE (2008) Görtler vortices in falkner-skan flows with suction and blowing. International Journal for Numerical Methods in Fluids 56(3):257-277. https://doi.org/10.1002/fld.1516

Mitsudharmadi H, Winoto SH, Shah DA (2006) Development of most amplified wavelength Görtler vortices. Physics of Fluids 18:014101. https://doi.org/10.1063/1.2160523

Otto SR, Denier JP (1993) The effect of crossflow on Görtler vortices. (CR-194897/R-94-19). NASA/ICASE Technical Report.

Ragab SA, Nayfeh AH (1981) Görtler instability. Physics of Fluids 24(8):1

Rogenski JK (2015) Influência do gradiente de pressão na transição em escoamentos sobre superfícies côncavas (PhD Thesis). São Carlos: Universidade de São Paulo.

Rogenski JK, Souza LF, Floryan JM (2013) Influence of curvature variations on the primary instability development in boundary layer flow. Presented at: 22nd International Congress of Mechanical Engineering; Ribeirão Preto, Brazil.

Rogenski JK, Souza LF, Floryan JM (2016a) Influence of pressure gradients on the evolution of the Görtler instability. AlAA Journal 54(9):2914-2919. https://doi.org/10.2514/1.J054658

Rogenski JK, Souza LF, Floryan JM (2016b) Non-linear aspects of Görtler instability in boundary layers with pressure gradient. Physics of Fluids 28(12):124107. https://doi.org/10.1063/1.4972241

Saric WS (1994) Görtler vortices. Ann Rev of Fluid Mechanics 26:379-409. https://doi.org/10.1146/annurev.fl.26.010194.002115

Schrader L, Brandt L, Zaki TA (2011) Receptivity, instability and breakdown of Görtler flow. J Fluid Mechanics 682:362-396. https:// doi.org/10.1017/jfm.2011.229

Sescu A, Thompson D (2015) On the excitation of Görtler vortices by distributed roughness elements. Theoretical and Computational Fluid Dynamics 29(1-2):67-92. https://doi.org/10.1007/s00162-015-0340-2

Souza LF (2003) Instabilidade centrífuga e transição para turbulência em escoamentos laminares sobre superfícies côncavas (PhD Thesis). São José dos Campos: Instituto Tecnológico de Aeronáutica.

Winoto SH, Durão DFG, Crane RI (1979) Measurements within Görtler vortices. J Fluids Engineering 101(4):517-520. https://doi. org/10.1115/1.3449021

Zhang H, Fasel H (1999) Spatial direct numerical simulation of Görtler vortices. Presented at: AlAA Fluid Dynamics Conference; Norfolk, USA. 